\title{
How to reconstruct dynamic cardiac PET data?
}

\author{
Piotr J. Slomka, PhD, ${ }^{\mathrm{a}, \mathrm{b}}$ Adam M. Alessio, PhD, ${ }^{\mathrm{c}}$ and Guido Germano, PhD ${ }^{\mathrm{a}, \mathrm{b}}$ \\ a Department of Medicine, Cedars-Sinai Medical Center, Los Angeles, CA \\ b David Geffen School of Medicine, UCLA, Los Angeles, CA \\ c Department of Radiology, University of Washington, Seattle, WA
}

Received Jul 11, 2016; accepted Jul 11, 2016

doi:10.1007/s12350-016-0608-y

\section{See related article, pp. 282-290}

\section{INTRODUCTION}

Myocardial blood flow measurements with PET have recently entered clinical practice with multiple clinical tools for quantification of myocardial blood flow and myocardial flow reserve. ${ }^{1}$ These measurements can be obtained without additional radiation or imaging from dynamic data on 3D PET/CT, complementing the information obtained from static and gated myocardial perfusion scans and providing additional prognostic information. ${ }^{2,3}$ At the same time, vendors have dramatically improved PET image reconstruction techniques, incorporating advanced image corrections, but the applications of these reconstruction techniques have been directed primarily to the oncological field. ${ }^{4}$ How will these recent advances in PET reconstruction affect quantification of myocardial blood flow?

In this issue of the Journal of Nuclear Cardiology, O'Doherty et $\mathrm{al}^{5}$ study the effect of iterative image reconstruction with resolution recovery on the quantification of myocardial blood flow. The particular technique used is a method implemented on GE 3D PET/CT scanner which includes built-in regularization implemented as Bayesian penalized likelihood. This recent algorithm has been used clinically in oncological imaging and has shown favorable characteristics compared to first-generation standard iterative technique, ordered subsets expectation maximization (OSEM) ${ }^{6}$ Although the authors study dynamic blood flow of 13-N-Ammonia,

Reprint requests: Piotr J. Slomka, Department of Medicine, Cedars-Sinai Medical Center, Los Angeles, CA; slomkap@cshs.org

J Nucl Cardiol 2017;24:291-3.

$1071-3581 / \$ 34.00$

Copyright (C) 2016 American Society of Nuclear Cardiology. the studies were performed for assessment of cardiac sarcoidosis, and therefore no stress blood flow data were analyzed. The authors conclude that the dynamic reconstruction with advanced techniques results in less noise than comparable reconstructions obtained with filtered back projection (FBP) and OSEM, and do not cause bias in the estimation of the input function. They also establish optimal parameters for this new reconstruction technique with respect to the noise.

The myocardial blood flow quantification has been performed only at resting condition by O'Doherty et al, and the question remains if the results can be extrapolated to a more typical application-measuring hyperemic myocardial blood flow and flow reservewhich are most relevant diagnostically. Nevertheless, it is certainly a timely initial study, since a new generation of reconstruction methods is being introduced in the clinical practice, and older algorithms such as FBP may not be even available on some of the new hardware platforms. It is therefore important to understand the benefits and limitations of the latest advances in image reconstruction as they relate to the established measurements of myocardial blood flow.

\section{WHY STILL FILTERED BACK PROJECTION?}

Early seminal PET studies, which established the quantitative models for myocardial flow estimation, used FBP and 2D PET. FBP provides a fast, direct solution with stable results. FBP is a linear, analytic algorithm, ensuring that on average image voxels are directly proportional to the measured activity. However, all PET (and SPECT) acquisitions have inherently noisy measurements due to count limitations, and this approach leads to relatively noisy images. Along with noise, FBP suffers from artifacts such as (1) positive or negative streaks arising from focal activity regions (potentially problematic for subdiaphragmatic activity that could cause streaks into the myocardium) and (2) negative values around low-activity regions. Myocardial blood flow measurements, especially 
early frames with extremely low-count densities, present specific challenges.

\section{WHY ITERATIVE RECONSTRUCTION METHODS?}

Because of its noise and artifacts, FBP is generally considered inferior to newer iterative methods. Originally, OSEM became a favored algorithm in PET because it offered rapid convergence in high-count regions. For oncology applications requiring hot feature detection, this was a favorable property as high-count lesions quickly appeared out of relatively low-count backgrounds. Unfortunately, OSEM has a slower convergence in low-count regions, making it less optimal for cold feature imaging. ${ }^{7}$ Considering that dynamic cardiac imaging requires a mix of good hot feature performance (especially for input function estimation) and cold feature performance (for defect zones along myocardium), the question regarding the validity of OSEM-type methods for cardiac dynamic flow measurements has not been fully settled. It is of particular importance that the area under the curve of the initial fast bolus inside left ventricle is measured accurately. A decade ago, when first-generation iterative techniques were introduced to clinical practice, some investigators found that the results were not interchangeable when compared to FBP. ${ }^{8}$ It has been demonstrated that iterative technique such as OSEM do not provide accurate image-derived input functions for cardiac PET studies because of bias in count estimation, ${ }^{9}$ although this bias can be potentially compensated by smoothing.

On the other hand, new reconstruction methods offer great potential for improved noise statistics ${ }^{8}$ and improved image fidelity. ${ }^{10}$ It has been demonstrated for cardiac dynamic applications that iterative techniques also yield improved image accuracy and less noise compared with FBP. ${ }^{11}$ The overall image appearance is of great importance for dynamic blood flow quantification because it will allow better delineation of the myocardial border and better detection of potential patient motion. The improved image quality will enable more reliable placement of the regions of interest for the blood and myocardium, and reduced reliance on manual contour adjustments and corrections. The improved overall repeatability of quantitative MBF measurements has been demonstrated with iterative reconstruction. ${ }^{12}$

\section{NEWER TECHNIQUES}

Although early studies of iterative reconstructions method have indicated some potential limitations, with respect to quantitative accuracy, newer implementations including resolution recovery and advanced regularization methods have been shown to mitigate some of these issues. For example, recently it has been shown that resolution recovery methods can reduce low-statistics bias in quantitative dynamic PET. ${ }^{13}$ Studies of optimal parameter setup for iterative techniques have been performed, recommending using more image updates performed with more iterations, but with less subsets, to reduce bias. ${ }^{14}$ Time-of-flight (TOF) methods combined with resolution recovery have been shown to improve quantification of dynamic cardiac PET compared to FBP and standard iterative reconstruction in a phantom study. ${ }^{15}$ Recently, the suitability of the TOF reconstruction with resolution recovery was studied in patients for $13-\mathrm{N}-\mathrm{Ammonia}$ blood flow quantification. ${ }^{16}$ The authors concluded that TOF allowed better visual image quality and contrast with TOF. TOF also improved the reproducibility of segmental flow estimates. While analytic TOF reconstruction methods have been proposed, it should be noted that the only commercial reconstruction methods for TOF data are iterative. The application of the new reconstruction techniques to the myocardial blood flow quantification is discussed in detail by Moody et al in a recent comprehensive review. ${ }^{17}$

\section{RUBIDIUM-82}

A note of caution is required in extrapolating these recent findings to dynamic blood flow quantification with Rubidium 82 (Rb-82), which is by far the most commonly used tracer in the United States for this application. Only one recent study reported application of TOF and resolution recovery for myocardial blood flow quantification with $\mathrm{Rb}-82 .{ }^{18}$ The authors found a considerable increase in the MBF values compared to standard OSEM and recommended further studies. The difficulties with Rb-82 may be related to the fact that resolution recovery and TOF techniques have been primarily developed for different tracers, and $\mathrm{Rb}-82$ requires special considerations due to longer positron path and the need for prompt gamma correction. ${ }^{19}$ Thus, a separate evaluation will be required to test the application of the new reconstruction methods for dynamic blood flow quantification with Rb-82.

Nevertheless, new iterative reconstruction techniques are becoming widely used in clinical studies of myocardial blood flow ${ }^{20-22}$ for various tracers. It will be important to note which exact methods and parameters have been applied when comparing the results from these studies and comparing the quantitative results and abnormality thresholds between these clinical studies. The comparative studies of different reconstruction techniques such as those presented by O'Doherty et al will provide valuable reference in this regard. 


\section{TAKE HOME MESSAGE}

Vendors are introducing several new reconstruction techniques, often replacing classic methods such as filtered back projection. The initial validation of these techniques is usually performed in the oncological applications. The new reconstruction techniques have the potential to improve the performance of dynamic cardiac imaging by providing lower noise images and better definition of the myocardium. These techniques are becoming widely used in clinical studies of myocardial blood flow. However, cardiac dynamic imaging has unique requirements due to the quantitative aspect of the measurements. Therefore, it is important to verify the application of these new methods for this application. Although some limitations have been demonstrated with the first-generation iterative techniques with respect to the quantitative accuracy, latest advances in noise regularization are likely to overcome these difficulties. Further validation studies are needed to better understand the use of these new techniques for the dynamic quantitative analysis.

\section{Disclosure}

None.

\section{References}

1. Nesterov SV, Deshayes E, Sciagrà R, Settimo L, Declerck JM, Pan $\mathrm{X}-\mathrm{B}$, et al. Quantification of myocardial blood flow in absolute terms using $82 \mathrm{Rb}$ PET imagingThe RUBY-10 study. JACC. 2014;7:1119-27.

2. Murthy VL, Naya M, Foster CR, Hainer J, Gaber M, Di Carli G, et al. Improved cardiac risk assessment with noninvasive measures of coronary flow reserve. Circulation. 2011;124:2215-24.

3. Ziadi MC, Dekemp RA, Williams KA, Guo A, Chow BJ, Renaud $\mathrm{JM}$, et al. Impaired myocardial flow reserve on rubidium-82 positron emission tomography imaging predicts adverse outcomes in patients assessed for myocardial ischemia. J Am Coll Cardiol. 2011;58:740-8.

4. Slomka PJ, Pan T, Germano G. Recent advances and future progress in PET instrumentation. Semin Nucl Med. 2016;46:5-19.

5. O'Doherty J, McGowan DR, Abreu C, Barrington S. Effect of Bayesian penalized likelihood reconstruction on $[13 \mathrm{~N}]-\mathrm{NH}_{3}$ rest perfusion quantification. J Nucl Cardiol. 2016. doi:10.1007/ s12350-016-0554-8.

6. Parvizi N, Franklin JM, McGowan DR, Teoh EJ, Bradley KM, Gleeson FV. Does a novel penalized likelihood reconstruction of 18F-FDG PET-CT improve signal-to-background in colorectal liver metastases? Eur J Radiol. 2015;84:1873-8.

7. Van Slambrouck K, Stute S, Comtat C, Sibomana M, van Velden $\mathrm{FH}$, Boellaard R, et al. Bias reduction for low-statistics PET: maximum likelihood reconstruction with a modified Poisson distribution. IEEE Trans Med Imaging. 2015;34:126-36.

8. Chen GP, Branch KR, Alessio AM, Pham P, Tabibiazar R, Kinahan $\mathrm{P}$, et al. Effect of reconstruction algorithms on myocardial blood flow measurement with 13N-Ammonia PET. J Nucl Med. 2007;48:1259-65.

9. Boellaard R, van Lingen A, Lammertsma AA. Experimental and clinical evaluation of iterative reconstruction (OSEM) in dynamic PET: Quantitative characteristics and effects on kinetic modeling. J Nucl Med. 2001;42:808-17.

10. Di Carli MF, Dorbala S, Meserve J, El Fakhri G, Sitek A, Moore SC. Clinical myocardial perfusion PET/CT. J Nucl Med. 2007;48:783-93.

11. Søndergaard HM, Madsen MM, Boisen K, Bøttcher M, Schmitz O, Nielsen TT, et al. Evaluation of iterative reconstruction (OSEM) versus filtered back-projection for the assessment of myocardial glucose uptake and myocardial perfusion using dynamic PET. Eur J Nucl Med Mol Imaging. 2007;34:320-9.

12. Efseaff M, Klein R, Ziadi MC, Beanlands RS, deKemp RA. Shortterm repeatability of resting myocardial blood flow measurements using rubidium-82 PET imaging. J Nucl Cardiol. 2012;19: 997-1006.

13. Walker MD, Asselin MC, Julyan PJ, Feldmann M, Talbot PS, Jones T, et al. Bias in iterative reconstruction of low-statistics PET data: benefits of a resolution model. Phys Med Biol. 2011;56: 931-49.

14. Jian $\mathrm{Y}$ and Carson RE. Effect of subsets on bias and variance in low-count iterative PET reconstruction. In 2013 IEEE Nuclear Science Symposium and Medical Imaging Conference (2013 NSS/ MIC). 2013:1-4.

15. Presotto L, Gianolli L, Gilardi MC, Bettinardi V. Evaluation of image reconstruction algorithms encompassing time-of-flight and point spread function modelling for quantitative cardiac PET: Phantom studies. J Nucl Cardiol. 2015;22:351-63.

16. Suda M, Onoguchi M, Tomiyama T, Ishihara K, Takahashi N, Sakurai M, et al. The reproducibility of time-of-flight PET and conventional PET for the quantification of myocardial blood flow and coronary flow reserve with $13 \mathrm{~N}$-ammonia. J Nucl Cardiol. 2016;23:457-72.

17. Moody JB, Lee BC, Corbett JR, Ficaro EP, Murthy VL. Precision and accuracy of clinical quantification of myocardial blood flow by dynamic PET: A technical perspective. J Nucl Cardiol. 2015;22:935-51.

18. Armstrong IS, Tonge CM, Arumugam P. Impact of point spread function modeling and time-of-flight on myocardial blood flow and myocardial flow reserve measurements for rubidium- 82 cardiac PET. J Nucl Cardiol. 2014;21:467-74.

19. Esteves FP, Nye JA, Khan A, Folks RD, Halkar RK, Garcia EV, et al. Prompt-gamma compensation in Rb-82 myocardial perfusion 3D PET/CT. J Nucl Cardiol. 2010;17:247-53.

20. Danad I, Raijmakers PG, Appelman YE, Harms HJ, de Haan S, van den Oever ML, et al. Hybrid imaging using quantitative H215O PET and CT-based coronary angiography for the detection of coronary artery disease. J Nucl Med. 2013;54:55-63.

21. Renaud J, Klein R, Hunter C, Ruddy T, DeKemp R. Test-retest repeatability of hypercapnea-stress Rb-82 PET myocardial blood flow is improved with dual-spillover correction in healthy normal subjects. J Nucl Med. 2016;57:146.

22. Thomassen A, Petersen H, Diederichsen ACP, Mickley H, Jensen LO, Johansen A, et al. Hybrid CT angiography and quantitative 15O-water PET for assessment of coronary artery disease: comparison with quantitative coronary angiography. Eur J Nucl Med Mol Imaging. 2013;40:1894-904. 